\title{
Contrast Agents
}

\section{OVERVIEW}

Contrast agents in general are exogeneous substances employed to alter natural tissue contrast. The motivation behind the use of contrast agents in MR imaging is to further enhance contrast between normal and diseased tissue types and indicate functionality of an organ. The contrast agents used in nuclear imaging techniques such as positron emission tomography (PET) and computed tomography (CT) provide a direct effect. On the other hand, in MR imaging, the effects of contrast agents are indirect and MR signal is not derived from the contrast agent itself but from its effect on the inherent proton relaxation times of the tissue in which it resides.

Effects of the contrast agent on relaxation times are expressed in terms of its relaxivities $\left(K_{1}, K_{2}\right)$ in liters per moles per sec (liter $\left./ \mathrm{mol} / \mathrm{sec}\right)$ and concentration of contrast agent $(C)$ in millimoles per liter ( $\mathrm{mmol} /$ liter):

$$
\frac{1}{T_{1}(C)}=\frac{1}{T_{1,0}}+K_{1} C
$$

and

$$
\frac{1}{T_{2}(C)}=\frac{1}{T_{2,0}}+K_{2} C
$$

where $T_{1,0}$ and $T_{2,0}$ are inherent longitudinal and transverse relaxation times, respectively. The relaxivity of the contrast agent is defined as the change in relaxation rate per unit concentration of contrast agent. One application of $T_{1}$-reducing contrast agents is to enhance blood vessels (Fig. B6.4.1).

In the healthy brain, the contrast agent cannot cross the blood/brain barrier and the signal enhancement occurs in the blood itself. In Fig. B6.4.1, it is shown that gray matter is also slightly enhanced. This is because it contains a small fraction of blood vessels. Another application of contrast agents is to detect the presence of tumors. Edge enhancement of tumors is seen because of the vascular content of the tumor. Although several contrast agents have been frequently used in MRI, currently an exogeneous contrast agent, Gd-DTPA (gadolinium diethylenetriamine pentaacetic acid) is the only MR contrast agent approved for human use in the United States. The focus in this unit will be given to the basic mechanisms of contrast agents in MR without going into their clinical applications.

\section{TECHNICAL DISCUSSION}

\section{Contrast Agents}

Several paramagnetic contrast agents such as Gd-DTPA, albumin-Gd-DTPA, Gd-DTPApolylysine, and dysprosium-DTPA (Dy-DTPA) have been widely used in MRI. Paramagnetic agents possess large magnetic moments, derived from their unpaired electrons, because single unpaired electrons have magnetic dipole moments 657 times larger than the magnetic dipole moment of a single, unpaired proton. In the absence of a paramagnetic contrast agent, the relaxation mechanism of a spin results from the dipolar interactions between nuclei as modulated by molecular rotational (tumbling) motion. In the presence of paramagnetic ions, the interaction between the magnetic dipole of the water proton and the magnetic dipole of the unpaired electron in the paramagnetic ion leads to $T_{1}$ shorten- 
A

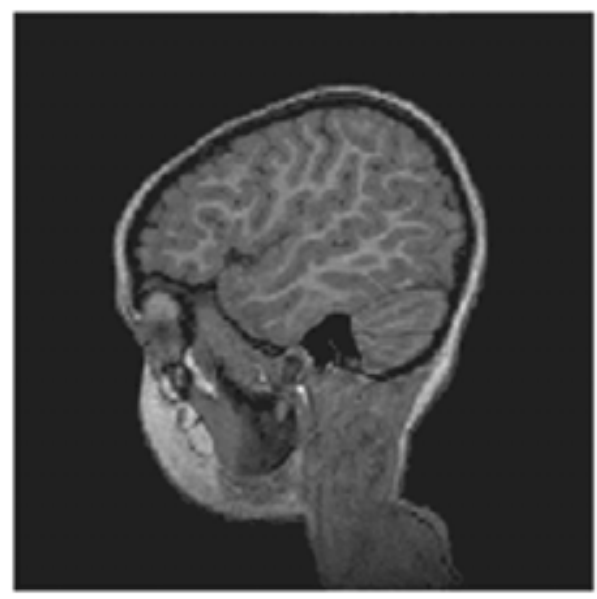

C

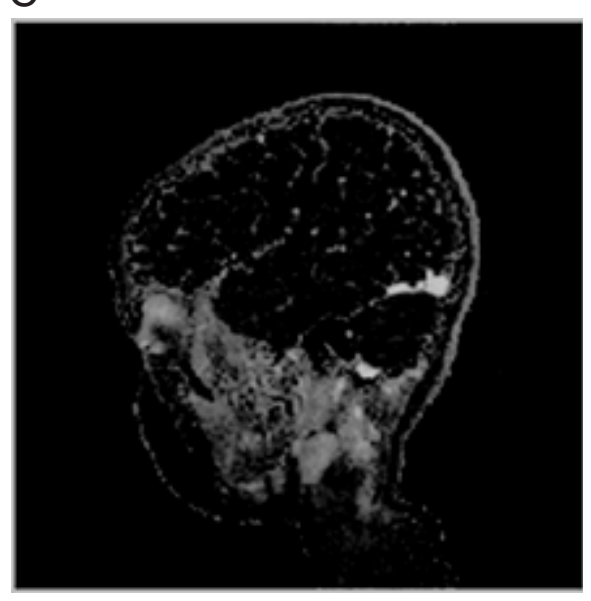

B

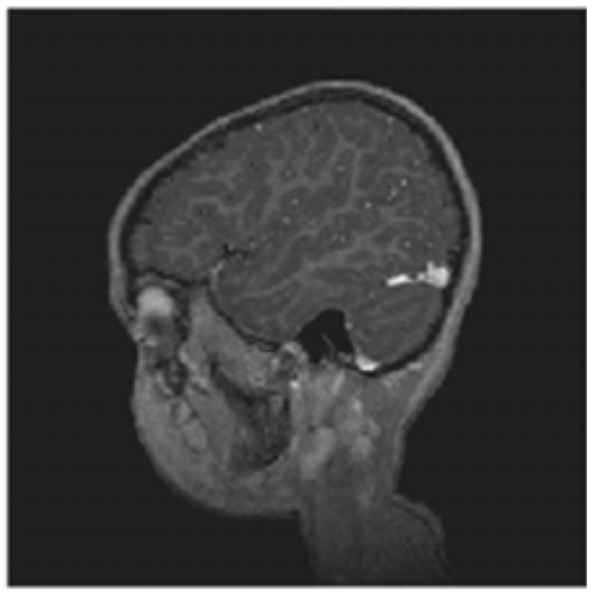

Figure B6.4.1 $T_{1}$ reduction effects of a contrast agent. (A) A single slice from a 3-D $T_{1}$-weighted data set before contrast agent was injected. (B) Image of the same slice after an intravenous injection of a $T_{1}$-shortening contrast agent. Since the $T_{1}$ of blood is shortened, the blood vessels light up, and are seen as bright spots in the image. Also, there is a loss of contrast between WM/GM because of the larger blood volume content of GM (hence, its signal increases more than that of WM, reducing contrast). (C) Subtraction of the first image from the second image depicts this signal increase predominantly in the blood vessels, but also from the blood-containing tissue itself. Only those voxels with a positive subtraction are shown; voxels with a negative subtraction are set to zero, leading to the isolated black dots in this image. The imaging parameters used were (for panels $A$ and $B$ ) $T_{R} / T_{E}=20 \mathrm{msec} / 6 \mathrm{msec}$, $\theta=25^{\circ}, T_{\mathrm{s}}=5.12 \mathrm{msec}, \mathrm{N}_{\mathrm{acq}}=1, \Delta x \times \Delta y \times \Delta z=0.9 \mathrm{~mm} \times 0.9 \mathrm{~mm} \times 2.33 \mathrm{~mm}, N_{x} \times N_{y} \times N_{z}=256 \times$ $224 \times 60, \tau_{\mathrm{RF}}=2.56 \mathrm{msec}, G_{\mathrm{SS}}=1.33 \mathrm{mT} / \mathrm{m}$.

ing. In addition, the tumbling paramagnetic ions with their large magnetic moments further augment the local magnetic variations experienced by the water protons, thereby causing them to dephase more rapidly (shortened $T_{2}$ ). The effects of paramagnetic contrast agents are both concentration- and magnetic field-dependent.

The relaxivity and concentration of a contrast agent are the two factors determining its effect on relaxation times. The relaxivity of a contrast agent can be defined as the change in relaxation rate $\left(\Delta R_{\mathrm{i}}\right)$ per unit concentration of contrast agent while the concentration is defined as the amount of contrast agent in a given volume. The relaxivities of the most

\section{B6.4.2}


commonly used agent, Gd-DTPA, have been found to be 4.5 liter $/ \mathrm{mmol} / \mathrm{sec}$ for $K_{1}$ and 5.0 liter $/ \mathrm{mmol} / \mathrm{sec}$ for $K_{2}$ in humans.

Consider a typical contrast-agent MR study where a single dose of Gd-DTPA (0.1 $\mathrm{mmol} / \mathrm{kg}$ ) is injected intravenously into an average adult with a body weight of $\sim 60 \mathrm{~kg}$. If we assume that the contrast agent is distributed evenly in the blood pool $(\sim 5.5 \mathrm{~kg})$, then the concentration of the contrast agent is $1.1 \mathrm{mmol} / \mathrm{liter}$. For inherent relaxation times of $1200 \mathrm{msec}\left(T_{1, \text { inherent }}\right)$ and $100 \mathrm{msec}\left(T_{2, \text { inherent }}\right)$ for venous blood, the effective relaxation times can be shown to be reduced to $173 \mathrm{msec}\left(T_{1, \text { eff }}\right)$ and $65 \mathrm{msec}\left(T_{2, \text { eff }}\right)$ for this particular concentration of the agent. Very quickly, much of the contrast agent is extravasated. Several minutes later, the concentration will be much lower, and $T_{1}$ values will significantly increase (if the concentration, $C$, becomes $0.55 \mathrm{mM}, T_{1}$ increases from $173 \mathrm{msec}$ to $302 \mathrm{msec})$.

The gadolinium ion $\left(\mathrm{Gd}^{3+}\right)$, the basis of Gd-DTPA, has 7 unpaired electrons and thereby possesses a very large magnetic moment. However, in its elementary form, this ion is toxic. Although the toxicity is reduced by binding $\mathrm{Gd}^{3+}$ to chelates, the price to pay for the reduced toxicity is the reduction in the number of binding sites available to water protons. Although Gd-DTPA is an extravascular and extracellular contrast agent, for normal brain imaging, it is confined in the vascular space by an intact blood-brain barrier.

A more recent contrast agent, Angiomark (EPIX), is the first gadolinium-based intravascular contrast agent to progress to human trials. Angiomark, unlike currently available extravascular and extracellular agents, binds strongly but reversibly to serum albumin in plasma. Findings in vivo indicate a strong, persistent plasma $T_{1}$ reduction with Angiomark for $1 \mathrm{hr}$ and a strong vascular enhancement on $T_{1}$-weighted MR images (Lauffer et al., 1998).

\section{Relaxivity and contrast enhancement}

In fast imaging, where $T_{\mathrm{R}}$ is much less than $T_{1}$, the signal for a $90^{\circ}$ flip angle scan is proportional to $T_{\mathrm{R}} / T_{1}$ and, in this regime, tissues with shorter $T_{1}$ have a higher signal than tissues with longer $T_{1}$, and the contrast is predominantly $T_{1}$-weighted.

Certain external agents can be introduced into specific targeted tissues where these agents act to reduce the $T_{1}$ of that tissue. Figure B6.4.1 demonstrates the $T_{1}$-shortening effect of one of these agents. Suppose that the targeted tissue (in the case shown in Fig. B6.4.1, the targeted tissue is blood) has similar NMR properties as its neighboring tissue, causing an inability to differentiate the two using any of the three contrast mechanisms discussed in UNIT B6.3, but contains a different signal response to the contrast agent. By delivering the $T_{1}$-shortening agent to the tissue of interest, the targeted signal is increased while the signal from the background remains the same when a $T_{1}$-weighted sequence is used. This increases the contrast between the two tissues, and, for this reason, such agents are commonly referred to as "contrast agents."

In general, the increase in relaxation rate after $T_{1}$ shortening is found to be directly proportional to the concentration $(C)$ of the contrast agent delivered to the tissue. If $T_{1,0}$ is the intrinsic $T_{1}$ of the tissue and $T_{1}(C)$ is its shortened value, it is found that:

$$
R_{1}(C) \equiv \frac{1}{T_{1}(C)}=\frac{1}{T_{1,0}}+K_{1} C \equiv R_{1,0}+K_{1} C
$$

where the constant of proportionality $K_{1}$ is called the longitudinal relaxivity ( $T_{1}$ relaxivity) with units of (mmol/liter $)^{-1} \mathrm{sec}^{-1}(1 \mathrm{mmol} / \mathrm{liter}$ is also written as $1 \mathrm{mM}$; millimolar), a property specific to the composition of the contrast agent. Figure B6.4.2A 
A

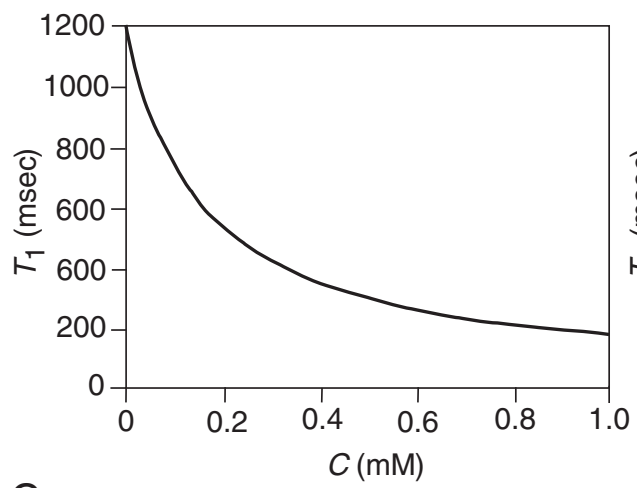

C

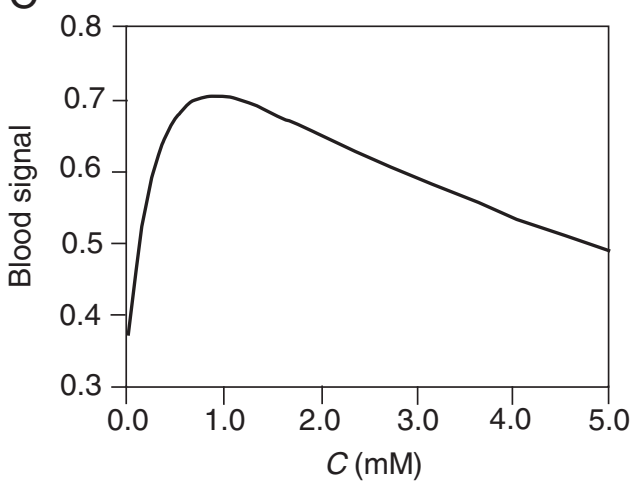

B

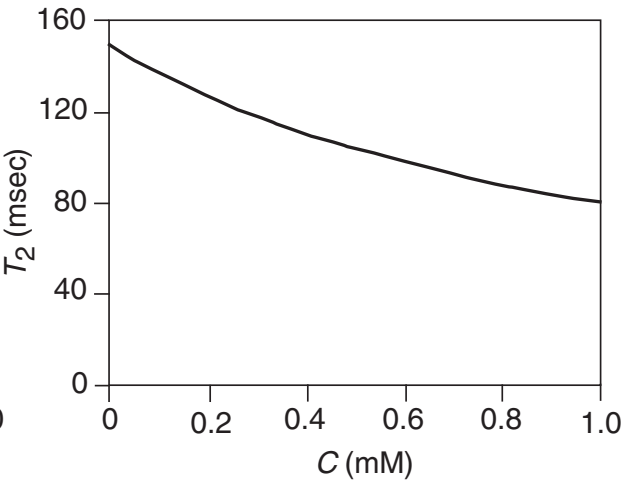

Contrast Agents

B6.4.4

Figure B6.4.2 $T_{1}$ and $T_{2}$ are plotted in $(\mathbf{A})$ and $(\mathbf{B})$, respectively, as functions of contrast agent concentration delivered to the tissue of interest. The contrast agent is supposed to have longitudinal and transverse relaxivities of $4.5 / \mathrm{mM} / \mathrm{sec}$ and $5.0 / \mathrm{mM} / \mathrm{sec}$, respectively. Notice that the relative change in $T_{1}$ for a given concentration is much larger than that for $T_{2}$ (C) Blood signal in relative units as a function of contrast agent concentration plotted for a spin echo acquisition with $600 \mathrm{msec} T_{\mathrm{R}}$ and $20 \mathrm{msec} T_{\mathrm{E}}\left(T_{1}\right.$ and $T_{2}$ of blood were taken to be $1200 \mathrm{msec}$ and $150 \mathrm{msec}$, respectively). Note that, as the concentration increases, the $T_{2}$-reduction effect takes over and causes a reduction of blood signal. In essence, an optimal concentration which maximizes the signal exists, in this case near $1 \mathrm{mM}$.

demonstrates the effect of a contrast agent in vivo on the $T_{1}$ of blood for which $\mathrm{T}_{1,0} \approx$ $1200 \mathrm{msec}$ at $1.5 \mathrm{~T}$.

In addition to shortening the $T_{1}$, these contrast agents also tend to shorten the $T_{2}$ of the tissue in a similar fashion to the $T_{1}$ shortening, i.e.:

$$
R_{2}(C) \equiv \frac{1}{T_{2}(C)}=\frac{1}{T_{2,0}}+K_{2} C \equiv R_{2,0}+K_{2} C
$$

where $K_{2}$ is the transverse relaxivity of the contrast agent. Figure B6.4.2B shows the effect of contrast agent dosage on the $T_{2}$ of blood (which has an intrinsic $T_{2}$ ranging from 100 msec for venous blood to $200 \mathrm{msec}$ for arterial blood). This concomitant decrease in $T_{2}$ tends to partly counterbalance the effects of shortened $T_{1}$. For many $T_{1}$-shortening contrast agents, the transverse and longitudinal relaxation rates are comparable in magnitude $\left(K_{1}\right.$ $\approx K_{2}$ ). Since $R_{2,0}>R_{1,0}$, a given increase in the concentration leads to a larger-magnitude change in $T_{1}$ and, hence, a larger-magnitude change in the signal due to this shortened $T_{1}$ effect than due to the $T_{2}$ reduction. It is only when $K_{2} C$ becomes comparable to $\mathrm{R}_{2,0}$ that the signal loss due to the $T_{2}$ shortening becomes significant and starts to overwhelm the 
enhanced $T_{1}$-weighted contrast. In fact, this cross-over point between signal loss due to $T_{2}$ shortening and signal increase due to $T_{1}$ shortening defines an optimal contrast agent dosage. New intravascular agents such as Angiomark (EPIX) tend to have a much higher $K_{1}$ (almost $25 / \mathrm{mM} / \mathrm{sec}$ ) and $K_{2}$ values similar to those of previous Gd-based agents.

A major clinical application of $T_{1}$-shortening contrast agents, at present, is intended for the improved detection of small lesions. Typically, lesions are best depicted in a $T_{2^{-}}$ weighted image before the contrast agent is injected. Despite being the most sensitive contrast mechanism, it is not possible for the $T_{2}$-weighted image to depict very small lesions which are averaged with neighboring tissue of comparable NMR tissue properties. The use of an intravenous injection of a $T_{1}$ shortening agent is indicated for such patients followed by a $T_{1}$-weighted scan (preferably a short $T_{\mathrm{E}}$, short $T_{\mathrm{R}}$, high-resolution gradientecho scan). Most lesions are found to have a rich blood supply, and the enhanced signal from the blood in the brain leads to improved lesion detection. Imaging of blood vessels is also dramatically improved using a contrast agent (see Chapter B7).

\section{LITERATURE CITED}

Lauffer, R.B., Parmelee, D.J., Dunham, S.U., et al. 1998. MS-325: Albumin-targeted contrast agent for MR angiography. Radiology 207:529-538.

\section{KEY REFERENCES}

Haacke, E.M., Brown, R.W., Thompson, M.R., and Venkatesan, R. 1999. Magnetic Resonance Imaging: Physical Principles and Sequence Design. John Wiley \& Sons, New York.

Contrast enhancement with $T_{1}$-shortening contrast agents is described in this text.

Lauffer et al., 1998. See above.

This article describes in detail the recent contrast agent, Angiomark.

Contributed by Azim Celik

General Electric Company

Milwaukee, Wisconsin

Weili Lin

The University of North Carolina at Chapel Hill

Chapel Hill, North Carolina

Signal, Noise, and

Contrast

B6.4.5 